\title{
MRI-based Diagnosis of Anterior Talofibular Ligament Injury
}

\author{
Lin $\mathrm{An}^{1}$ and Yaowen $\mathrm{Yan}^{2}$ \\ ${ }^{1}$ Department of Trauma Orthopaedics, Ningbo No. 6 Hospital, Ningbo, China \\ ${ }^{2}$ Department of Radiology, Xinchang People's Hospital, Zhejiang, China
}

\begin{abstract}
The aim of this study was to explore the value of magnetic resonance imaging (MRI) in the diagnosis of anterior talofibular ligament injury. Eighty-two patients, diagnosed with anterior talofibular ligament injury by pathological examination after operation, were included. MRI showed that there were 15 cases (18.29\%) of complete rupture, 50 cases (60.98\%) of tear and 12 cases (14.63\%) of avulsion fracture. The accuracy of MRI in the diagnosis of anterior talofibular ligament injury was $93.90 \%$ (77 cases). Compared with the pathological examination after operation, the difference was statistically insignificant $(p=0.059)$. The accuracy of MRI in the diagnosis of anterior talofibular ligament injury is high, and it has important clinical value in the early diagnosis of anterior talofibular ligament injury.
\end{abstract}

Key Words: Anterior talofibular ligament, Injury, Magnetic resonance imaging (MRI), Diagnosis.

How to cite this article: An L, Yan Y. MRI-based Diagnosis of Anterior Talofibular Ligament Injury. J Coll Physicians Surg Pak 2021; 31(11):1369-1371.

The injury of anterior talofibular ligament was the most common type among lateral collateral ligament injury of ankle joint. ${ }^{1}$ If patients with anterior talofibular ligament injury are not treated in time, the incidence of lateral ankle instability and traumatic arthritis increases, causing ankle dysfunction, which affect the overall patients' quality of life. Therefore, early diagnosis and treatment plays an important role in improving the prognosis of patients with anteriortalofibularligamentinjury.

Missed diagnosis rate of physical examination for diagnosis of anterior talofibular ligament injury is high. In clinic, physical examinations, combined with imaging examinations, are used for diagnosis of anterior talofibular ligament injury. X-ray, MRI (magnetic resonance imaging) and ultrasound were the main imaging methods with the development of technology. Among them, MRI is a medical imaging diagnosis technology with high resolution, zero damage, no pain and no radiation hazard. However, price of MRI examination is high.

The soft tissue was destroyed after the injury of anterior talofibular ligament, and it could not be clearly displayed by general imaging techniques. MRI has high resolution for soft tissue and has evident advantages in diagnosis of suspected ATFL injury. ${ }^{2}$ Ankle MRI scanning can help, diagnose anterior talofibular ligament (ATFL) injury. ${ }^{3}$ Bright rim sign is seen on conventional MRI in the diagnosis of ATFL rupture. $^{4}$

Correspondence to: Yaowen Yan, Department of Radiology, Xinchang People's Hospital, Zhejiang, China

E-mail: xuujgr@163.com

Received: April 29, 2021; Revised: July 20, 2021;

Accepted: August 13, 2021

DOI: https://doi.org/10.29271/jcpsp.2021.11.1369
The objective of this study was to explore the value of MRI in the diagnosis of anterior talofibular ligament injury.

This study was approved by the Research Ethical Committee of Ningbo No. 6 Hospital, China. Eighty-two patients, diagnosed with anterior talofibular ligament injury by pathological examination after operation from February 2018 to October 2020, were included. The inclusion criteria were $>18$ years of age, proven to be anterior talofibular ligament injury by pathological examination after operation. Osteoarthritis, ankle infection and tumor were excluded.

The clinical manifestation was limited internal rotation of the ankle joint. The ankle joint was swollen and accompanied by pain; and there was a history of trauma. All patients were examined by MRI before surgery. Posture of patients during MRI were in supine position, natural straightening of lower extremities and flexion of ankle joint in 20. Siemens Magnetom Avanto Syngo 1.5T superconducting MRI scanner was used to scan T1WI, T2WI and SPIR sequences in axial and coronal positions, respectively. The repetition time was $3000 \mathrm{~ms}$, echo time was $90 \mathrm{~ms}$, scanning layer number was 20 layers, scanning layer thickness was $3.0 \mathrm{~mm}$, scanning layer spacing was $0.3 \mathrm{~mm}$, matrix was $512 \times 512$, and the scanning visual field was 200 $\mathrm{mm}$. The main plane of anterior talofibular ligament and its surrounding structure was axial and coronal position of MRI. TIWI could clearly display the anatomical structure of ankle joint, T2WI and SPIR could clearly show the pathological changes.

The classification criteria for MRI diagnosis of anterior talofibular ligament injury were: (i) If the continuous change and shortening occurred in the whole broken ligament disruption, it was 
judged as complete rupture; (ii) If the SPIR sequence signals increased and the ligament thickened, it was judged as tear; (iii) If the severely damaged ligament was completely interrupted, the joint cavity around the anterior talofibular ligament showed the presence of effusion, and an avulsion fracture occurred, it was judged as accompanied by an avulsion fracture; (iv) If MRI showed that the anterior talofibular ligament was normal, it was judged as no damage.

SPSS version 25 were used for data analysis. The measurement data in accordance with the normal distribution were represented by mean $\pm \mathrm{SD}$, and the counting data were represented by $\mathrm{n}(\%)$, and the Fisher's Exact test was used for statistic comparison. Statistical significance was set at $p<0.05$.

Eighty-two patients, 37\45.12\% men and 45 (54.88\%) women, were included, aged 20-63 years with an average age of $36.66 \pm$ 6.52 years. Forty-nine (59.76\%) patients were injured in the left ankle joint and $33(40.24 \%)$ in the right ankle joint. The causes of injury include traffic injury $(n=40,48.78 \%)$, sports injury $(n=21$, $25.61 \%)$, direct impact injury $(n=15,18.29 \%)$, and sprained injury $(n=6,7.32 \%)$.

All cases were diagnosed with anterior talofibular ligament injury by operative and pathological examination after operation, complete rupture in 15 cases (18.29\%), tear in 54 cases $(65.85 \%)$ and avulsion fracture in 13 cases (15.85\%).

MRI showed that there were 15 cases (18.29\%) of complete rupture, 50 cases $(60.98 \%)$ of tear and 12 cases (14.63\%) of avulsion fracture. The accuracy of MRI in the diagnosis of anterior talofibular ligament injury was 93.90\% (77 cases). Compared with the pathological examination after operation, the difference was statistically insignificant $(p=0.059)$.

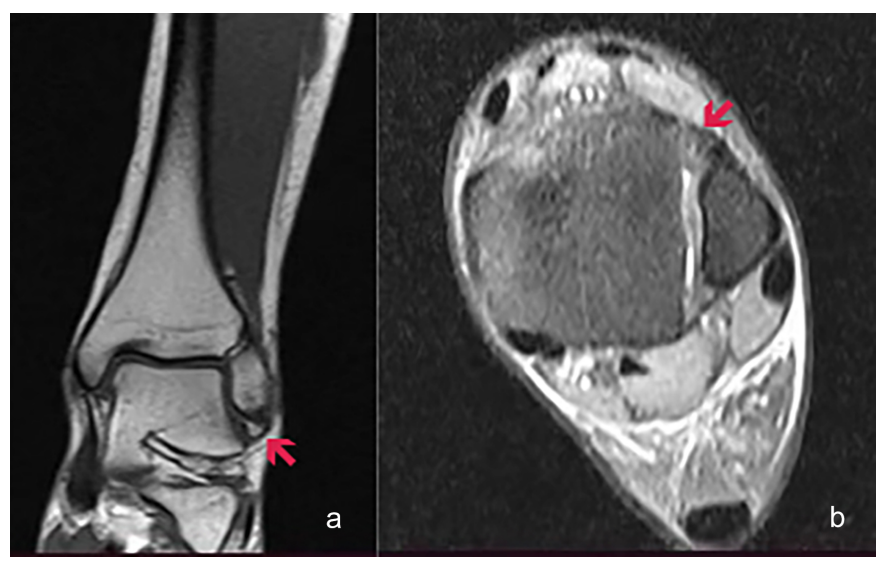

Figure 1: MRI scan of ankle joint. (a) Coronal plane of ankle joint, (b) Sagittal plane of ankle joint, showing thickened torn ATF ligament.

Figure 1 showed a classical case in a 36-year lady, who came to the hospital because of pain and limited movement of the sprained left ankle joint. MRI plain scan showed the injury of the left anterior talofibular ligament. MRI showed a tear, as increased SPIR sequence signal, and thickened ligaments.

The common type of anterior talofibular ligament injury was complete rupture or avulsion. The complete rupture showed separation and shortening of the fault end, bleeding, edema and anterior talo-peroneal ligament injury with avulsion fracture. The MRI findings of anterior talofibular ligament tear injury were interruption of ligament hypointensity in T2WI and high signal intensity appears, the outline of ligament was rough and the appearance changed clearly. ${ }^{5}$

The results of this study showed 15 cases (18.29\%) of complete rupture, 50 cases $(60.98 \%)$ of tear, and 12 cases $(14.63 \%)$ of avulsion fracture were diagnosed by MRI. The accuracy of MRI in the diagnosis of anterior talofibular ligament injury was $93.90 \%$ (77 cases), compared with the pathological examination after operation, the difference was statistically insignificant. Moreover, one study showed that MRI is detected in ATFL injury with $100 \%$ sensitivity and specificity. ${ }^{6}$

MRI can clearly display the anatomical characteristics and pathological changes of ligaments, and it has high sensitivity and specificity for the detection of ankle ligament injury. Hydrogen atoms and edematous fluid showed high signal intensity in ligaments on MRI imaging, and ligament was swelled and thicken due to bleeding and edema. Thus, MRI has high accuracy in the diagnosis of anterior talofibular ligament injury.

MRIhasthe characteristics ofmultiplanarimaging and high resolution, which has obvious advantages in examining the structure and tissue of ligaments. The accuracy of MRI in the diagnosis of anterior talofibular ligament injury is high, and it has important clinical value in the early diagnosis of anterior talofibular ligament injury.

\section{PATIENTS' CONSENT:}

Written informed consents were obtained from all patients, who participated in thisstudy.

\section{CONFLICT OF INTEREST:}

All authors declared no conflict of interest.

\section{AUTHORS' CONTRIBUTION:}

LA: Conception, design of the work, acquisition, analysis, interpretation of data for the work, drafting work.

YY: Conception, critical review of intellectual content and final approval of the work.

\section{REFERENCES}

1. Casado I, Vallejo R, Iglesias ML, Nuo FS, Lobo CC. Association between anterior talofibular ligament injury and ankle tendon, ligament, and joint conditions revealed by magnetic resonance imaging. Quant Imaging Med Surg 2021; 11(1):84-4. doi: 10.21037/qims-20-5.

2. Xu Y, He L, Han Y, Duan D, Ouyang L. Evaluation of 3dimensional magnetic resonance imaging (3D MRI) in diagnosing anterior talofibular ligament injury. Med Sci Monit 2021; 27:e927920-1-e927920-6. doi: 10.12659/MSM. 927920.

3. Cao S, Wang C, Ma X, Wang X, Huang J, Zhang C, et al. Reliability and validity of different ankle MRI scanning planes for the anterior talofibular ligament injury diagnosis: A cadaveric study. J Orthop Surg Res 2019; 14(1):69-77. doi: org/10.1186/s13018-019-1102-4

4. Basha M, Eldib DB, Zaitoun M, Ghandour TM, Algazzar HY. 
The added diagnostic value of the bright rim sign to conventional MRI assessment of anterior talofibular ligament disruption. Acad Radiol 2020; S1076-6332(20): 30281-6. doi.org/10.1016/j.acra.2020.05.005.

5. Yao W, Zhang Y, Zhang L, Zhou J, Zhang Y, Zheng X, et al. MRI features of and factors related to ankle injuries in asymptomatic amateur marathon runners. Skeletal Radiol 2021:50(1):87-95. doi: 10.1007/s00256-020-03530-9.

6. Azni KK, Saini SM, Nasir N, Bahari N. Correlation of clinical examination, magnetic resonance imaging and surgical findings in diagnosing ankle joint ligament injuries. European J Molecul Clin Med 2020; 7(2):1474-84. 